\title{
The Outcome of Monochorionic Diamniotic Twins Discordant at 11 to $13^{+6}$ Weeks' Gestation
}

\author{
Yaniv Zipori, ${ }^{1}$ Karen Reidy, ${ }^{1}$ T. Gilchrist, ${ }^{2}$ Lex W. Doyle, ${ }^{1,3,4}$ and Mark P. Umstad ${ }^{1,2}$ \\ ${ }^{1}$ Department of Obstetrics and Gynecology, University of Melbourne, Royal Women's Hospital, Melbourne, Victoria, \\ Australia \\ ${ }^{2}$ Division of Maternity Services, Royal Women's Hospital, Melbourne, Victoria, Australia \\ ${ }^{3}$ Neonatal Services, Royal Women's Hospital, Melbourne, Victoria, Australia \\ ${ }^{4}$ Clinical Sciences, Murdoch Children's Research Institute, Melbourne, Victoria, Australia
}

\begin{abstract}
Background: Monochorionic diamniotic (MCDA) twin pregnancies are associated with adverse perinatal outcome. Intertwin discordances at the time of nuchal translucency (NT) screening may have a value in the prediction of fetal loss or twin-to-twin transfusion syndrome. We aimed to determine the ability of intertwin NT and crown rump length (CRL) discordances among MCDA twins to predict adverse outcomes. Material and Methods: All MCDA twins with a documented routine ultrasound at 11 to $13^{+6}$ weeks' gestation, and known pregnancy outcome between August 2003 and August 2012 were included. Receiver operating characteristic curves were used to determine the ideal NT and CRL discordances cut-off points that maximized the ability to predict adverse outcome, which was defined as any of: death of one or both twins, twin-to-twin transfusion syndrome, or estimated fetal weight or birth weight discordances $\geq 25 \%$. Results: Of the 89 cases, 20 (22.5\%) had at least one adverse outcome. NT discordance was more discriminatory of adverse outcome than was CRL discordance. The optimal values for predicting any adverse outcomes for NT were $>23.7 \%$ and for CRL $>3.5 \%$. The positive predictive values for NT $(52.4 \%)$ and CRL $(29.8 \%)$ screening were relatively low; however, the lack of either NT or CRL discordances was more reassuring, with negative predictive values of $86.8 \%$ and $86.4 \%$, respectively. Conclusions: NT discordance is more predictive for adverse fetal outcome in MCDA twins than CRL discordance. Neither NT nor CRL discordance are likely to modify the intensive monitoring required for these very high-risk pregnancies.
\end{abstract}

Keywords: crown rump length, discordance, monochorionic diamniotic twins, nuchal translucency, twin-to-twin transfusion syndrome

Although the majority of monochorionic diamniotic (MCDA) twins have a successful pregnancy outcome, the presence of hemodynamic instability in MCDA twins, secondary to their unequal placenta sharing, and the coexistence of interfetal anastomoses (rescue transfusion) may endanger the entire pregnancy. Twin-to-twin transfusion syndrome (TTTS), selective fetal growth restriction (sFGR), death in utero of one or both fetuses, and birth weight discordance are known contributors to adverse outcomes in MCDA twins (Acosta-Rojas et al., 2007; Gratacós et al. 2012; Lewi et al., 2008).

Several ultrasound features (nuchal translucency [NT] discordance and crown rump length [CRL] discordance in early and late first trimester), either separately or in combination, have been postulated as indirect predictors of placental circulatory imbalance. Kagan et al. (2007) have shown that NT discordance over $20 \%$ between the MCDA twin pair was present in about $25 \%$ of cases, and in this group, the risk of developing complications (TTTS or fetal death of one or more twins) is $30 \%$. If the NT discordance is less than $20 \%$, the risk of such complications is less than $10 \%$ (Kagan et al., 2007). On the contrary, Casasbuenas et al. (2008) found no significant correlation with the subsequent development of TTTS when comparing MCDA twins with increased versus normal NT thickness (Casasbuenas et al., 2008). Though previous studies suggested a role for CRL discrepancy in the routine 11 to $13^{+6}$ weeks' scan as a marker for subsequent development of fetal loss

RECEIVED 22 June 2016; ACCEPTED 14 September 2016. First published online 21 October 2016.

ADDRESS FOR CORRESPONDENCE: Mark P. Umstad, Department of Obstetrics and Gynecology, Royal Women's Hospital, 20 Flemington Rd, Parkville VIC 3052, Australia. E-mail: Mark.Umstad@thewomens.org.au 
of one or both twins, from both TTTS and sFGR (Bhide et al., 2009; El Kateb et al., 2007; Memmo et al., 2012), the recent large STORK study has clearly stated that CRL discordance is of poor predictive value for adverse perinatal outcome in MCDA twins, and should not be used routinely to identify MCDA twins at high risk of adverse perinatal outcome (D'Antonio et al., 2013). Currently, there is no reliable dominant positive predictive marker for the development of MCDA related complications.

The aim of this study was to determine the ability of NT and CRL discordances among MCDA twins to predict adverse fetal outcomes.

\section{Material and Methods}

\section{Study Design}

This was a retrospective cohort study, which was approved as an audit by the Human Research Ethics Committee at The Royal Women's Hospital (RWH), Melbourne, Australia.

\section{Study Population}

All twin gestations delivered at RWH, Australia over a 9year period from August 2003 to August 2012 were initially screened to identify the participants for the study. Of the 126 identified selected MCDA twins, 89 cases with documented measurements of NT and CRL on ultrasound between 11 and $13^{+6}$ weeks' gestation, and known pregnancy outcome were selected for the study. Exclusion criteria included known lethal anomalies (including chromosomal abnormalities) at the time of $11-13^{+6}$ week scan, loss of one or both twins prior to the $11-13^{+6}$ week scan, and altered chorionicity findings on placental histology.

The estimated date of delivery was derived from the reported last menstrual period and was confirmed by the earliest ultrasound result available from maternal medical records. When an early CRL discrepancy existed between the twins, the largest fetus was used for determination of estimated due date. In Australia, evaluation of fetal CRL and NT between 11 and $13^{+6}$ weeks is part of the routine ultrasound examination in multiple pregnancies, and performed by ultrasonologists or ultrasonographers accredited by the Royal Australian and New Zealand College of Obstetricians and Gynaecologists (RANZCOG).

The percentage discrepancy for NT was determined as the percentage difference relative to the lower value for NT. The percentage discrepancy for CRL was determined as the percentage difference relative to the larger value for CRL.

Monochorionicity was determined by ultrasound demonstration of a single placental mass with the presence of a typical T-sign, and confirmed after delivery by placental histology. A routine fetal morphology scan was carried out between 18 and 20 weeks' gestation. As per the RWH preference policy, MCDA twins had fortnightly ultrasound assessments until delivery, commencing at 16 weeks of gestation, to detect pregnancy complications.

\section{TABLE 1}

Maternal and Fetal Characteristics of the Cohort and Ultrasound Discordance Values

\begin{tabular}{ll}
\hline Characteristics & $n=89$ \\
\hline Age: years - mean (SD) & $31.2(5.3)$ \\
Body Mass Index (weight/height ${ }^{2}$ ) - mean (SD) & $24.4(4.4) ; n=72$ \\
Assisted reproduction - $n(\%)$ & $16 / 87(18.4)$ \\
Nulliparous - $n(\%)$ & $42(47.2)$ \\
Nuchal thickness difference: \% - median (IQR) & $15.4(6.5,29.7)$ \\
Crown rump length difference: \% - median (IQR) & $3.6(1.6,6.8)$ \\
Gestational age at delivery (weeks) - mean (SD) & $34.1(3.3)$ \\
TTTS - $n(\%)$ & $13(14 \%)$ \\
\hline Note: IQR = interquartile range (25th and 75th centiles); TTTS = twin-to- \\
$\quad$ twin transfusion syndrome.
\end{tabular}

\section{Data Collection}

Data were collected from the combined maternal, fetal, and neonatal clinical records at our hospital. When women delivered elsewhere, their general practitioner or obstetrician was contacted to collect the clinical details and outcomes.

\section{Outcomes of Study}

The major adverse fetal outcome measure of the study was defined as a combined outcome comprising any of death of one or both fetuses after the first trimester NT screening scan (including after birth), the development of TTTS as defined by Quintero et al. (1999), the presence of major fetal anomalies not detected at the time of the 11 to $13^{+6}$ week scan, estimated fetal weight (EFW) discordance of $\geq 25 \%$ on ultrasound at 28 weeks' gestation, or a birth weight discordance of $\geq 25 \%$. We were also interested in the outcome of TTTS alone.

\section{Statistical Analysis}

Data were analyzed using SPSS for Windows, version 21. Continuous variables are described as mean $(S D)$, or median (interquartile range [IQR; 25th-75th centiles]) if data were skewed. Receiver operating characteristic (ROC) curves were plotted for both NT and CRL discordance to determine the cut-off point that maximized the ability to predict the adverse outcomes. The area under the curve (AUC) and its 95\% confidence intervals (CIs) were used to determine the statistical significance of each of NT and CRL discordance at predicting adverse outcomes. Using the optimal cut-points identified from the ROC curves, sensitivity, specificity, and positive and negative predictive values were determined for each variable.

\section{Results}

The maternal characteristics of the MCDA pregnancies included in the study are shown in Table 1. Of note, only $18 \%$ of women conceived with assisted reproductive techniques. The median NT and CRL discordance values for the study cohort were $15.4 \%$ and $3.6 \%$, respectively.

Of the 89 cases, $20(22.5 \%)$ had at least one adverse outcome; $16(18 \%)$ had at least one fetal loss after $13^{+6}$ weeks, 


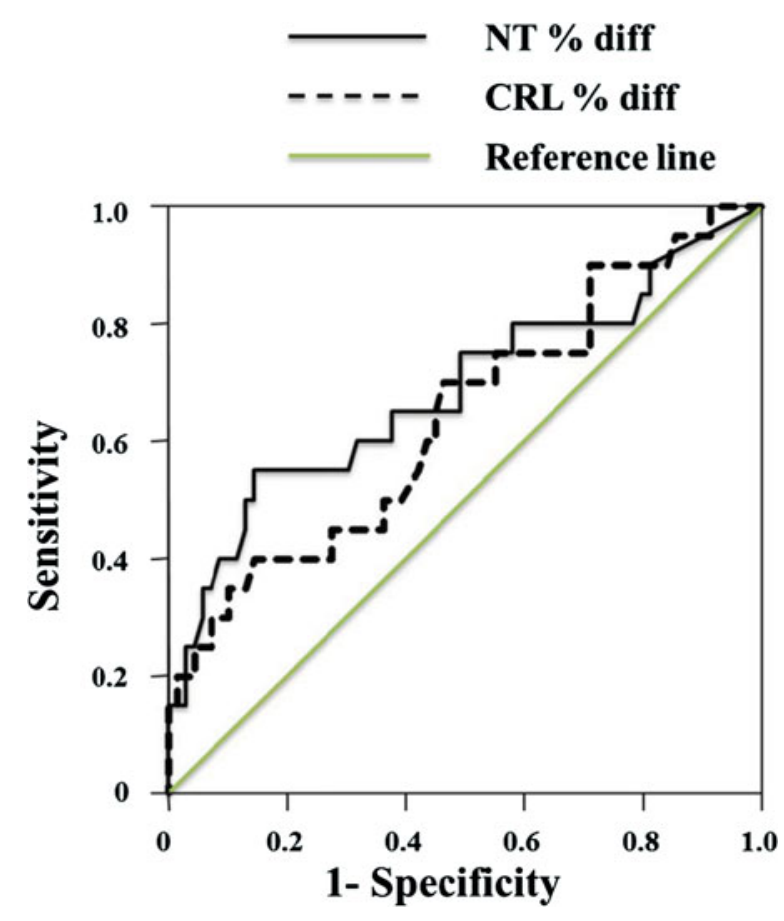

FIGURE 1

(Colour online) Receiver operating characteristics (ROC) curves for the prediction of combined adverse perinatal outcomes in MCDA twins $^{\dagger}$ for both NT and CRL discordance.

Note: ${ }^{\dagger}$ Any of: fetal loss of at least one of the twins, TTTS, estimated fetal weight discordance $\geq \mathbf{2 5} \%$, birth weight discordance $\geq \mathbf{2 5 \%}$. NT = nuchal translucency; CRL = crown-rump length.

13 (14\%) experienced TTTS, two (2\%) had EFW discordance $\geq 25 \%$, and seven ( $8 \%$ ) had birth weight discordance $\geq 25 \%$.

In 12 of the 16 pregnancies with a fetal loss, both fetuses died - seven cases of TTTS, three spontaneous miscarriages unrelated to TTTS, one case secondary to a later diagnosis of cardiac anomaly of both twins (NT discordance $>$ $20 \%$ ), and one case of trisomy 21 (no NT discordance). In the remaining four cases, there was loss of a single twin; all were due to complications associated with TTTS. All of the fetal losses of both twins were under 20 weeks of gestation. Of the 13 TTTS cases, three had laser intervention for stage 2 (two cases) and stage 3 (one case) and one case had amniodrainage for stage 3 TTTS. The remaining cases were either followed conservatively for early stage TTTS (two cases) or had termination of pregnancy or spontaneous miscarriage (seven cases).

Both cases of ultrasound EFW $\geq 25 \%$ discordance also had birth-weight discordance $\geq 25 \%$. Of the seven pregnancies with birth-weight discordance, $\geq 25 \%$, only one twin subsequently died after birth from a major anomaly (a tracheoesophageal fistula that was suspected only in the third trimester secondary to late non-visualization of the stomach).
The ROC curves for NT and CRL discordances for the combined adverse perinatal outcome are shown in Figure 1. NT discordance with an AUC of 0.68 [95\% CI, 0.54, 0.83; $p$ value .012] was a better predictor than CRL discordance with an AUC of 0.64 [95\% CI, 0.50, 0.78; $p$ value .074] for an adverse outcome. The optimal values for predicting any adverse outcomes derived from the ROC curves for NT were $>31.1 \%$ and for CRL $>3.5 \%$. These were also the optimum values for predicting TTTS (Figure 2), although neither variable was a strong predictor of TTTS on the ROC curves; NT discordance, AUC 0.66 [95\% CI, 0.49, 0.83; $p$ value .064]; CRL discordance, AUC 0.60 [95\% CI, 0.43, 0.76; $p$ value .27]. The sensitivities, specificities, and the positive and negative predictive values of NT and CRL discordances as screening tests in predicting either any adverse outcome or the individual TTTS complication are shown in Table 2. NT discordance had better diagnostic ability than CRL for either any adverse outcome or the individual TTTS outcome, particularly with higher positive predictive values, which were substantially higher than the prevalence for both outcomes.

\section{Discussion}

Unequal placental sharing in MCDA twins increased the risk of adverse pregnancy outcome. The evolution of the unique TTTS and sFGR forms in MCDA twins may endanger the entire pregnancy (Acosta-Rojas et al., 2007; Gratacós et al. 2012; Lewi et al., 2008). Twin anemia polycythemia sequence (TAPS) is yet another less common, recently described, adverse outcome of MCDA twins; thus, uniform criteria still need to be clearly established. Nevertheless, TAPS can be suspected antenatally on the basis of absence of ultrasounds signs of oligohydramnios and polyhydramnios, Doppler abnormalities showing an increased peak systolic velocity in the middle cerebral artery (MCAPSV) in the donor twin (suggestive of fetal anemia) and a decreased MCA-PSV in the recipient twin (suggestive of polycythemia) (Slaghekke et al., 2010).

The risk of developing at least one adverse fetal outcome in MCDA pregnancies in this study was $22 \%$. Increased NT discordance detected by ultrasound at 11 to $13^{+6}$ weeks was a better diagnostic tool at predicting adverse perinatal outcomes than was CRL discordance.

The use of antenatal ultrasound for prediction of potential MCDA complications has received considerable attention in recent years. The widespread routine implementation of ultrasound screening at 11 to $13^{+6}$ weeks provides an opportunity to study potential hemodynamic instability in MCDA twins. The hope is to identify those pregnancies that require increased surveillance, and possibly early intervention, to improve the overall outcome and prognosis. Increased surveillance likely will include more frequent ultrasound scans to allow early detection of TTTS or sFGR and earlier intervention by laser in a timely fashion. On the 
TABLE 2

Diagnostic Accuracy of NT and CRL Discordance for any Adverse Outcome, and for TTTS Alone

\begin{tabular}{lllllll}
\hline & Sensitivity (\%) & Specificity (\%) & PPV (\%) & NPV (\%) & p value & Prevalence \%* \\
\hline For any adverse outcome & & & & & & \\
$\quad$ NT $>31.1 \%$ & 55.0 & 85.5 & 52.4 & 86.8 & $<.001$ & 22.5 \\
$\quad$ CRL $>3.5 \%$ & 70.0 & 53.6 & 30.4 & 86.0 & .063 & 22.5 \\
For TTTS alone & & & & & & \\
$\quad$ NT $>31.1 \%$ & 53.8 & 81.1 & 33.3 & 90.9 & .007 & $14.9(13 / 87)$ \\
CRL $>3.5 \%$ & 69.2 & 49.35 & 19.6 & 90.2 & .20 & $14.9(13 / 87)$ \\
\hline
\end{tabular}

Note: *Prevalence of any adverse fetal outcome or TTTS. PPV = positive predictive value; NPV = negative predictive value; NT = nuchal translucency; $C R L=$ crown-rump length.

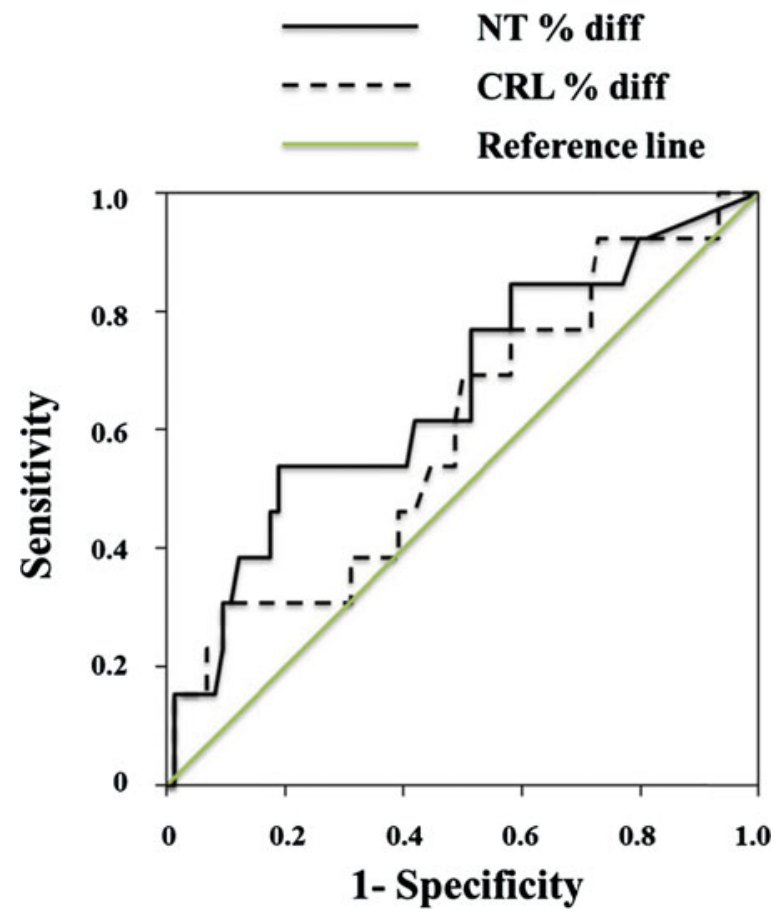

FIGURE 2

(Colour online) Receiver operating characteristics (ROC) curves for the prediction of TTTS in MCDA twins ${ }^{\dagger}$ for both NT and CRL discordance.

Note: ${ }^{\dagger}$ Any of: fetal loss of at least one of the twins, TTTS, estimated fetal weight discordance $\geq \mathbf{2 5 \%}$, birth weight discordance $\geq \mathbf{2 5 \%}$. NT = nuchal translucency; CRL = crown-rump length.

contrary, frequent use of ultrasound and early intervention might also affect clinical care negatively (e.g., cost of ultrasound, women having termination of pregnancy), whereby actions and decisions are made on the basis of false positive prediction or conversely misplaced reassurance.

The prognosis of untreated stages II to IV TTTS is bleak, with reported antenatal loss rates ranging from 70-100\%, particularly if it develops prior to 26 weeks (Berghella \& Kaufmann, 2001; Gul et al., 2003). Fetoscopic laser photocoagulation has been demonstrated to substantially improve outcomes. A survival rate in excess of $75 \%$ of at least one twin and major reduction in the risk of longterm major neurological impairment has been repeatedly shown (Salomon et al., 2010; Senat et al., 2004). In our co- hort, all four cases of advanced TTTS who underwent in utero therapy resulted in the successful live birth of one of the twins.

Intertwin NT or CRL discordancy in MCDA twins offers the first window of opportunity to assess the potential for later MCDA complications. Despite the controversy in the literature, there is a general understanding that increased NT discordances among MCDA twins mandate a close surveillance for potential complications (Kagan et al., 2007; Salomon et al., 2010). In the majority of cases, surveillance involves more frequent ultrasound examinations than the proposed fortnight ultrasounds for MCDA twins without NT discordances.

Unlike NT discordance, emerging evidence does not support a significant role for CRL discordance as a major predictive marker of complications in MCDA pregnancies (D’Antonio et al., 2013; Johansen et al., 2014). We found that NT discordance $>31.1 \%$ was associated with significant combined adverse outcomes, as well as with the individual TTTS complication. The overall positive predictive value of NT screening was rather low in predicting any adverse outcome at $52.4 \%$. However, the lack of NT discordance can be seen as reassuring, with a negative predictive value of $86.8 \%$. Both of these values need to be considered relative to the prevalence of $22 \%$, so neither NT or CRL discordance are likely to modify the intensive monitoring required for these very high-risk pregnancies. CRL discordance appears to have less value in predicting adverse outcome in MCDA twins than NT discordance. This was also seen in the recent STORK study, in which the predictive accuracy of CRL discordance was poor for fetal loss $<24$ weeks, perinatal mortality, EFW discordance, birth weight discordance, or preterm birth $<34$ weeks (D'Antonio et al., 2013). CRL discordance $\geq 3.5 \%$ in our study group was not significantly predictive of potential adverse outcomes.

A limitation of this study was the small size of the cohort, with only 89 out of the early 126 MCDA twin pregnancies having NT and CRL measurements, as well as known pregnancy outcome.

In conclusion, NT discordance $>31.3 \%$ identifies MCDA twin pregnancies at higher risk of potential complications. CRL discordance is of limited value in predicting any adverse outcome. Absence of substantial NT and CRL 
concordance, however, may be somewhat reassuring for prospective parents and their healthcare providers.

\section{References}

Acosta-Rojas, R., Becker, J., Munoz-Abellana, B., Ruiz, C., Carreras, E., Gratacos, E., Catalunya and Balears Monochorionic Network. (2007). Twin chorionicity and the risk of adverse perinatal outcome. International Journal of Gynecology and Obstetrics, 96, 98-102.

Berghella, V., \& Kaufmann, M. (2001) Natural history of twin-twin transfusion syndrome. Journal of Reproductive Medicine, 46, 480-484.

Bhide, A., Sankaran, S., Sairam, S., Papageorghiou, A. T., \& Thilaganathan, B. (2009). Relationship of intertwin crownrump length discrepancy to chorionicity, fetal demise and birth-weight discordance. Ultrasound in Obstetrics \& Gynecology, 34, 131-135.

Casasbuenas, A., Wong, A. E., \& Sepulveda, W. (2008). Nuchal translucency thickness in monochorionic multiple pregnancies: Value in predicting pregnancy outcome. Journal of Ultrasound in Medicine, 27, 363-369.

D’Antonio, F., Khalil, A., Dias, T., Thilaganathan, B., Southwest Thames Obstetric Research Collaborative. (2013). Crownrump length discordance and adverse perinatal outcome in twins: Analysis of the Southwest Thames Obstetric Research Collaborative (STORK) multiple pregnancy cohort. Ultrasound in Obstetrics \& Gynecology, 41, 621-626.

El Kateb, A., Nasr, B., Nassar, M., Bernard, J. P., \& Ville, Y. (2007). First-trimester ultrasound examination and the outcome of monochorionic twin pregnancies. Prenatal Diagnosis, 27, 922-925.

Gratacós, E., Ortiz, J. U., \& Martinez, J. M. (2012). A systematic approach to the differential diagnosis and management of the complications of monochorionic twin pregnancies. Fetal Diagnosis and Therapy, 32, 145-155.

Gul, A., Aslan, H., Polat, I., Cebeci, A., Bulut, H., Sahin, O., \& Ceylan, Y. (2003). Natural history of 11 cases of twin-twin transfusion syndrome without intervention. Twin Research, 6, 263-266.
Johansen, M. L., Oldenburg, A., Rosthøj, S., Cohn Maxild, J., Rode, L., \& Tabor, A. (2014). Crown-rump length discordance in first trimester: A predictor of adverse outcome in twin pregnancies? Ultrasound in Obstetrics \& Gynecology, 43, 277-283.

Kagan, K. O., Gazzoni, A., Sepulveda-Gonzalez, G., Sotiriadis, A., \& Nicolaides, K. H. (2007). Discordance in nuchal translucency thickness in the prediction of severe twin-totwin transfusion syndrome. Ultrasound in Obstetrics \& Gynecology, 29, 527-532.

Lewi, L., Jani, J., Blickstein, I., Huber, A., Gucciardo, L., Van Mieghem, T., ... Deprest, J. (2008). The outcome of monochorionic diamniotic twin gestations in the era of invasive fetal therapy: A prospective cohort study. American Journal of Obstetrics \& Gynecology, 199, 514.e1-514.e1-8.

Memmo, A., Dias, T., Mahsud-Dornan, S., Papageorghiou, A. T., Bhide, A., \& Thilaganathan, B. (2012). Prediction of selective fetal growth restriction and twin-to-twin transfusion syndrome in monochorionic twins. BJOG, 119, 417421.

Quintero, R. A., Morales, W. J., Allen, M. H., Bornick, P. W., Johnson, P. K., \& Kruger, M. (1999). Staging of twin-twin transfusion syndrome. Journal of Perinatology, 19, 550-555.

Salomon, L. J., Ortqvist, L., Aegerter, P., Bussieres, L., Staracci, S., Stirnemann, J. J., ... Ville, Y. (2010). Long-term developmental follow-up of infants who participated in a randomized clinical trial of amniocentesis vs laser photocoagulation for the treatment of twin-to-twin transfusion syndrome. American Journal of Obstetrics \& Gynecology, 203, 444.e1-e7.

Senat, M. V., Deprest, J., Boulvain, M., Paupe, A., Winer, N., \& Ville, Y. (2004). Endoscopic laser surgery versus serial amnioreduction for severe twin-to-twin transfusion syndrome. New England Journal of Medicine, 351, 136-144.

Slaghekke, F., Kist, W. J., Oepkes, D., Pasman, S. A., Middeldorp, J. M., Klumper, F. J., ... Lopriore, E. (2010). Twin anemia-polycythemia sequence: Diagnostic criteria, classification, perinatal management and outcome. Fetal Diagnosis and Therapy, 27, 181-190. 